\title{
Research Square \\ Risk Factors of ICU-Acquired Weakness in Critical III Patients with ECMO Support: A Retrospective Study
}

xinyi chen

Zhejiang University School of Medicine

xiong lei

Zhejiang University School of Medicine

xin xu

Zhejiang University School of Medicine

Man Huang ( $\nabla$ huangman@zju.edu.cn )

Zhejiang University School of Medicine Second Affiliated Hospital https://orcid.org/0000-0002-08422254

Research

Keywords: ECMO, ICU-acquired weakness, Adrenaline, Infection

Posted Date: April 20th, 2021

DOI: https://doi.org/10.21203/rs.3.rs-415136/v1

License: (c) (1) This work is licensed under a Creative Commons Attribution 4.0 International License.

Read Full License 


\section{Risk factors of ICU-acquired weakness in critical ill patients with ECMO support: a retrospective study}

Xinyi Chen M.M.1, Xiong Lei M.M.1, Xin Xu M.M.2, Man Huang M.D.1

1. 1) Department of General Intensive Care Unit, The Second Affiliated Hospital of Zhejiang University School of Medicine, Hangzhou, China. E-mail: chenxinyi0323@zju.edu.cn.

2. 2) Department of General Intensive Care Unit, The Second Affiliated Hospital of Zhejiang University School of Medicine, Hangzhou, China. E-mail: 13868043096@163.com.

3. 3) Department of General Intensive Care Unit, The Second Affiliated Hospital of Zhejiang University School of Medicine, Hangzhou, China. E-mail: zyeyzhicu@zju.edu.cn.

Corresponding author:

Man huang, Department of General Intensive Care Unit, The Second Affiliated Hospital of Zhejiang University School of Medicine, Hangzhou, China. E-mail: huangman@zju.edu.cn 


\begin{abstract}
Background: Muscle weakness is exceedingly common in critical illness patients and is well described. However, information on the risk factors of muscle weakness in critically ill patients who received extracorporeal membrane oxygenation (ECMO) support are lacking. Our study aims to clarify the risk factors of ICU-acquired weakness (ICU-AW) in ECMO patients to help early recognize potential weakness.
\end{abstract}

Methods: We conducted a retrospective study of critical ill patients who used ECMO during their ICU stay. ICU-AW was diagnosed at the time when patients discharged and had a muscle strength sum score $<48$ out of a maximal score of 60 . Baseline characters and therapy details were collected from the case report form and inspection report. Univariable analysis and logistic analysis were used to analyze clinical characteristics of individuals.

Results: 63 patients receiving ECMO support were divided to ICU-AW group and no ICU-AW group according to the Medical Research Council (MRC) score when discharged. A total of 50 of the 63 patients were diagnosed with ICU-AW. On logistic regression analysis, the use of adrenaline apparently increased the odds of developing ICU-AW ( $\mathrm{OR}=6.8,95 \% \mathrm{CI} 1.19-38.92, \mathrm{p}=0.032)$, and it was independent of all other established risk factors for ICU-AW. Meanwhile, the occurrence of infection during ECMO support significantly increased the risks of ICU-AW $(\mathrm{OR}=7.3,95 \%$ CI 1.28$41.24, \mathrm{p}=0.025$ ) and was independently associated with the outcome of ICU-AW. Other factors such as mechanical ventilation duration, steroid use and neuromuscular blockade did not differ significantly between the groups in our analysis.

Conclusion: Infection during ECMO support and use of adrenaline were found independently associated with the development of ICU-AW.

Keywords: ECMO, ICU-acquired weakness, Adrenaline, Infection 


\section{Introduction}

ECMO is increasingly being used worldwide in recent years as a rescue therapy for critical ill patients[1]. Though ECMO has almost become standard care as a therapy in critical ill patients when less invasive measures have failed, it is potentially associated with serious complications, such as bleeding, infection, acute kidney injury and neuromuscular complications, which should be considered and weighted[2].

According to previous studies, ICU-AW can occur in critical ill patients several days after ICU admission and muscle loss can exceed $10 \%$ on the first week in ICU[3,5]. Muscle strength and mass reduction are common and $43 \%$ of critical ill patients have suffered muscle strength decrease[4,5].It leads difficulty in weaning off mechanical ventilation, which in turn increase the risk of ICU-AW and diagram dysfunction[6,7]. Moreover, ICU-AW increases long-term and short-term mortality rate and decreases the life quality when discharged hospital[8].

ICU-AW is a usual complication in critical ill patients with a prevalence of $43 \%$ (interquartile range 25-75\%) [5,9]. Patients with ECMO support always suffers more risk factors of ICU-AW such as prolonged mechanical ventilation duration, deep sedation and paralyzed, and long-time of immobility, they are more likely to suffer ICU-AW. Thus, we need to pay more attention to distinguish those are more vulnerable to muscle weakness.

Few studies to review the risk factors of ICU-AW in patients following ECMO support. The goal of our study is to find the risk factors of ICU-AW in ECMO support individuals. We herein collect clinical information of our patients and analyze their characteristics, hoping to provide some clinical reference for our colleagues. We hypothesize that ICU-AW is frequent in patients following ECMO support and it might relate to infection during ECMO support and the use of adrenaline but is not associated with ECMO days, the use of neuromuscular block agents and use of steroid. 


\section{Methods}

Study design and setting

We conducted a retrospective study of critical ill patients who use ECMO during their ICU stay. Patients were recruited from the general ICU of the second affiliated hospital of Zhejiang University, between the years 2017 and 2020 .

Patients

Patients over 18 years old who had received ECMO therapy during ICU stay were eligible for inclusion criteria in the study. Exclusion criteria were patients who had been proven or suspected neurological impairment, using ECMO less than 24 hours, severe head or spinal cord injury and pregnant woman.

Limb Muscle Strength

Muscle strength was assessed by physical and occupational therapists on hospital discharge. The strength of muscle of included patients were assessed by MRC score, using a scale from 0 to 5 . ICU-AW was diagnosed at the time when a patient is awake and attentive who had a muscle strength sum score $<48$ out of a maximal score of 60.

Processes

Following enrollment, demographic data was collected immediately which included gender, age, BMI score, SOFA score, APACHE II score, reasons for admitted to ICU. Therapy details were recorded as well, time and depth of sedation, nutrition and glycaemic control condition, ventilator settings and duration, drug details and rehabilitation information were included. The RASS score was recorded every hour when patients were under sedation, excepting the patients were undergoing rescue, physical therapy or undergoing some clinical treatment such as bronchoscopy and intubation. The actual calories and protein intake were recorded in patients used enteral nutrition except 1 patient in ICU-AW group used parenteral nutrition. The goal calories were $25-30 \mathrm{kcal} / \mathrm{kg}$ and the goal protein intake was $1.2-2.0 \mathrm{~g} / \mathrm{kg}$. ECMO settings (types of ECMO, rotating speed, gas speed, blood speed, fraction of inspiration O2) and ventilator settings (highest PEEP, ventilator mode) were collected 1 day after ECMO initiation. Complications during ECMO support were also recorded from the case report form and inspection report.

Statistical analysis

Continuous variables were expressed as median (median \pm interquartile range) or mean (mean 
standard deviation). Categorical variables were expressed as frequency. Statistical analysis was performed using SPSS, tests exercised were pearsons test, fishers exact test, non-parametric test and logistic analysis; statistical significance was assumed at the five percent level. Statistical analyses were performed with SPSS version 23 .

\section{Results}

Over 3 years of the study period, 85 patients who had used ECMO were assessed for eligibility. Among the 85 patients, 22 patients presented exclusion criteria and 63 patients were included in the final analysis (Fig 1). A total of 50(79\%) patients were diagnosed with ICU-AW when discharged.

\section{Baseline characteristics of the study population}

Baseline characteristics of the population are described in Table 1. VV-ECMO and VA-ECMO were most commonly used, there were 31 patients using VV-ECMO support and 29 patients using VA-ECMO support. The number of users of different types of ECMO was shown in Fig 2. Severe pneumonia and acute myocardial infarction were most common reasons for ICU admission. Notably, APACHE II score and SOFA score were comparable between 2 groups, patients with ICU-AW had higher APACHE II score (14(9-19.5) versus 19(16-25.5), $\mathrm{p}=0.005)$ and SOFA score (7.5(6-10.5) versus $11(8-13), p=0.005)$ compared with no ICU-AW.

\section{Therapy details during intensive care unit stay}

Table 2 demonstrated the therapy details of the study population. The median RASS score during ICU stay differed between 2 groups $(-2.3(-3.0--1.4)$ versus $-2.9(-3.6--2.3), \mathrm{p}=0.013)$. The ICU-AW group underwent sedation time at a median of 211.6 hours whereas no ICU-AW group underwent a median time of 132.3 hours, but did not differ significantly in 2 groups. Meanwhile, average glucose levels and insulin infusion was similar between 2 groups, including the percentage of patients reached goal calories and protein intake.

Patients in ICU-AW group experience longer ventilation time during ECMO operation (3.70(2.3-5.5) versus 5.80(3.3-10.9), $\mathrm{p}=0.037)$. The median duration of mechanical ventilation did not differ significantly between 2 groups. Meanwhile, characteristics of mechanical ventilation did not differ significantly between 2 groups, including the mode, settings of ventilator, the highest PEEP level and the percentage of tracheotomy. Mechanical ventilation duration of no ICU-AW 
group was shorter compared with ICU-AW but did not statistically significant. The median mechanical ventilation days from start of ventilation until initiation of ECMO was $0.24 \mathrm{~d}$ and $0.39 \mathrm{~d}$ and there were not comparable between 2 groups.

Notably, the use of adrenaline during ICU stay was more common in ICU-AW group than in no ICU-AW group. $88 \%$ patients with ICU-AW received adrenaline compared with $38.5 \%$ of patients with no ICU-AW (5(38.5\%) versus 44(88\%), p=0.001). Other risk factors such as steroid use and use of neuromuscular blockade agents did not differ significantly in our analysis. Moreover, there is no difference whether patients participate in rehabilitation exercises.

\section{ECMO management}

ECMO parameters including types of ECMO, rotating speed, gas speed, blood speed, fraction of inspiration $\mathrm{O} 2$, cannulation and complications during ECMO operation were showed in Table3 They were not comparable except the occurrence of infection and liver and kidney dysfunction during ECMO support. As compared to no ICU-AW group, ICU-AW group had higher percentage of infection during ECMO support (4(30.8\%) versus 36(72\%), $\mathrm{p}=0.015)$. The percentage of awake ECMO and the median length of ECMO duration appeared no significant difference in our research.

\section{Logistic regression analysis of ICU-AW}

Table 4 showed the logistic regression of ICU-AW. Based on the univariable analysis, the following variables were included in the multivariable analysis: APACHE II score, average RASS score, infection during ECMO use, mechanical ventilation time during ECMO use and the use of adrenaline. Among all these variables, the use of adrenaline was associated more than 6.8 folds increase in the odds of developing ICU-AW. The occurrence of infection during ECMO operation significant increase the risk of develop ICU-AW (OR=7.3,95\% CI 1.28-41.24, $\mathrm{p}=0.025)$.

\section{Discussion}

With the advancements in modern intensive care medicine, mortality of critical ill patients has decreased, however, at the cost of a growing incidence of ICU-AW[10]. ICU-AW is known to have detrimental effects on both short-term and long-term clinical outcomes, identifying those who at high risk of developing ICU-AW is important[8,11].In our research, we show in a population of critical ill patients that the use of adrenaline and occurrence of infection are closely associated with 
the development of ICU-AW when discharged.

We showed in a population of critical ill patients with ECMO support, there was higher proportion of adrenaline use in ICU-AW group, the use of adrenaline significantly increased the risk of developing ICU-AW. Recently also reported findings identified that the use of catecholamine associated closely with the occurrence of ICU-AW[12]. Other adverse effects of adrenaline had been observed such as pulmonary edema, thrombus formation, gastrointestinal hypoperfusion, stimulation of bacterial growth and skeletal muscles apoptosis[13].

Previous researches indicated that the use of adrenaline was independently associated with the occurrence of cardiac events[14]. Skeletal muscle also a kind of muscle tissues, it is likely that adrenaline may affect skeletal muscle in some way. In animal models, catecholamines were found to induce myocyte apoptosis in the soleus muscle[15]. Moreover, isoproterenol and adrenaline produced most significant skeletal muscle toxicity of all adrenergic agents[13]. Catecholamines function by stimulation of $\alpha$ - and $\beta$-adrenergic receptors. In skeletal muscle, it expresses $\beta 1$ - and $\beta 2$-adrenergic receptor and $\beta 2$-adrenergic receptor is the predominant isoform[16]. So $\beta 2$-adrenergic receptors respond more strongly to adrenaline and it may account for the muscle toxicity of adrenaline. This indicates that adrenaline, maybe an independent risk factors associated with the ICU-AW, having a direct effect on the development of muscle weakness in critical ill patients.

In our analysis, infection during ECMO operation was an independent risk factor for the development of ICU-AW. Sepsis and multiple organ failure were considered associated with neuromuscular complications. Approximately $47 \%-70 \%$ of patients admitted to ICU for sepsis or multiple organ failure developed axonal motor-sensory damage with 1-3 weeks[17]. Apart from nerve injury, muscle mass reduction and decreased muscle strength were important features of ICUAW[6]. Increased loss of protein and decreased muscle protein synthesis might account for the decreased muscle strength and muscle mass reduction[6,18]. The potential mechanisms of protein reduction may through ubiquitin-proteasome system and autography[10,19]. Muscle satellite cell injury was found in sepsis-induced muscle weakness and might account for the failure of muscle regeneration in critical ill patients[20,21].

Though there is no effective way to rescue muscle weakness in patients with severe infection[6]. Clinical research found that obese patients are less likely to have ICU-AW[22]. In animal models, ketone diester was found to attenuate skeletal muscle atrophy and inflammation-induced catabolism, 
which demonstrating anti-catabolic effects of ketone bodies in muscle atrophy[23]. Clinical study also revealed that ketogenic diet has the potential to improve neurological outcomes for patients with various traumatic injuries[24]. Thus, we hypotheses that ketone bodies may be beneficial to prevent the development of muscle weakness in critical ill patients. Muscle satellite cells are found to experience injury in sepsis-induced muscle weakness, which result in regeneration damage and influence muscle strength recovery. In animal models, stem cell therapy is found to restore mitochondrial function and improving muscle fiber tension[21].

Other risk factors such as mechanical ventilation time during ECMO operation differed between 2 groups but was not independently associated with the development of ICU-AW. The use of steroid and the use of neuromuscular blocking agents didn't show apparent significant in our research. We primary revealed the risk factors in critical ill patients using ECMO support and found the use of adrenaline and infection during ECMO support independently associated with the development of ICU-AW.

This study has a number of limitations. First, our major limitation is the small number of included patients. Secondly, the data set is examined retrospectively, which limits the comparison to other studies. More clinical researches are needed to identify the risk factors and clinical characteristics of ICU-AW in patients with ECMO support to help early identify ICU-AW.

\section{Conclusion}

In our analysis, infection during ECMO support and use of adrenaline were found independently associated with the development of ICU-AW. 


\author{
Abbreviations \\ ECMO: Extracorporeal membrane oxygenation \\ ICU: Intensive care unit \\ ICU-AW: ICU-acquired weakness \\ OR: Odds ratio
}

\title{
Declarations:
}

Ethics approval and consent to participate: Our research was approved by the ethics committee of the Second Affiliated Hospital of Zhejiang University School of Medicine.

Consent for publication: Yes.

Availability of data and materials: The datasets used and analyzed during the current study are available from the corresponding author on reasonable request.

Competing interest: None.

Funding: No funding.

Author contributions: XC designed the research, analyzed the characteristics of the data and drafted the manuscript. XL helped design the research. XL and XX collected the clinical data and helped check the manuscript. MH supervised the research process, provided clinical reference, and revised the paper. All authors read and approved the final manuscript.

Acknowledgements: Thanks for all the staff of ICU for providing useful reference and study details of the study. 


\section{References}

1. Serpa Neto A, Schmidt M, Azevedo LCP, Bein T, Brochard L, Beutel G, et al. Associations between ventilator settings during extracorporeal membrane oxygenation for refractory hypoxemia and outcome in patients with acute respiratory distress syndrome: a pooled individual patient data analysis: Mechanical ventilation during ECMO. Intensive Care Med. 2016;42:1672-84. doi 10.1007/s00134-016-4507-0.

2. White A, Fan E. What is ECMO? Am J Respir Crit Care Med. 2016;193:P9-10. doi: 10.1164/rccm.1936P9.

3. Puthucheary ZA, Rawal J, McPhail M, Connolly B, Ratnayake G, Chan P, et al. Acute Skeletal Muscle Wasting in Critical Illness. JAMA. 2013;310:1591. doi: 10.1001/jama.2013.278481.

4. Dres M, Dubé B-P, Mayaux J, Delemazure J, Reuter D, Brochard L, et al. Coexistence and Impact of Limb Muscle and Diaphragm Weakness at Time of Liberation from Mechanical Ventilation in Medical Intensive Care Unit Patients. 2017;195:10. doi: 10.1164/rccm.20160203670C.

5. Fan E, Cheek F, Chlan L, Gosselink R, Hart N, Herridge MS, et al. An Official American Thoracic Society Clinical Practice Guideline: The Diagnosis of Intensive Care Unit-acquired Weakness in Adults. Am J Respir Crit Care Med. 2014;190:1437-46. doi: 10.1164/rccm.201411-2011ST.

6. Vanhorebeek I, Latronico N, Van den Berghe G. ICU-acquired weakness. Intensive Care Med. 2020;46:637-53. doi: 10.1007/s00134-020-05944-4.

7. Combes A, Schmidt M, Hodgson CL, Fan E, Ferguson ND, Fraser JF, et al. Extracorporeal life support for adults with acute respiratory distress syndrome. Intensive Care Med [Internet]. 2020 [cited 2020 Nov 19]; Available from: http://link.springer.com/10.1007/s00134-02006290-1. doi: 10.1007/s00134-020-06290-1.

8. Aerde NV. Five-year impact of ICU-acquired neuromuscular complications: a prospective, observational study. Intensive Care Med. 2020;46(6):1184-1193. doi: 10.1007/s00134-02005927-5.:10.

9. Harnisch L-O, Riech S, Mueller M, Gramueller V, Quintel M, Moerer O. Longtime Neurologic Outcome of Extracorporeal Membrane Oxygenation and Non Extracorporeal Membrane Oxygenation Acute Respiratory Distress Syndrome Survivors. J Clin Med. 2019 12;8(7):1020. doi: 10.3390/jcm8071020

10. Friedrich O, Reid MB, Van den Berghe G, Vanhorebeek I, Hermans G, Rich MM, et al. The Sick and the Weak: Neuropathies/Myopathies in the Critically III. Physiological Reviews. 2015;95:1025-109. doi: 10.1152/physrev.00028.2014.

11. Thille AW, Boissier F, Muller M, Levrat A, Bourdin G, Rosselli S, et al. Role of ICU-acquired weakness on extubation outcome among patients at high risk of reintubation. Crit Care. 2020;24:86. doi: 10.1186/s13054-020-2807-9. 
12. Wolfe KS, Patel BK, MacKenzie EL, Giovanni SP, PohIman AS, Churpek MM, et al. Impact of Vasoactive Medications on ICU-Acquired Weakness in Mechanically Ventilated Patients. Chest. 2018;154:781-7. doi: 10.1016/j.

13. Dünser MW, Hasibeder WR. Sympathetic Overstimulation During Critical Illness: Adverse Effects of Adrenergic Stress. J Intensive Care Med. 2009;24:293-316. doi: 10.1177/0885066609340519.

14. Schmittinger CA, Torgersen C, Luckner G, Schröder DCH, Lorenz I, Dünser MW. Adverse cardiac events during catecholamine vasopressor therapy: a prospective observational study. Intensive Care Med. 2012;38:950-8. doi: 10.1007/s00134-012-2531-2.

15. Iwai-Kanai E, Hasegawa K, Araki M, Kakita T, Morimoto T, Sasayama S. $\alpha$ - and $\beta$ Adrenergic Pathways Differentially Regulate Cell Type-Specific Apoptosis in Rat Cardiac Myocytes. Circulation. 1999 20;100(3):305-11. doi: 10.1161/01.cir.100.3.305.

16. Steiner JL, Johnson BR, Hickner RC, Ormsbee MJ, Williamson DL, Gordon BS. Adrenal stress hormone action in skeletal muscle during exercise training: An old dog with new tricks? Acta Physiol (Oxf). 2021 Jan;231(1): e13522. doi: 10.1111/apha.13522.

17. Jolley SE, Bunnell AE, Hough CL. ICU-Acquired Weakness. Chest. 2016 Nov;150(5):11291140. doi: 10.1016/j.chest.2016.03.045.

18. Klaude M, Mori M, Tjäder I, Gustafsson T, Wernerman J, Rooyackers O. Protein metabolism and gene expression in skeletal muscle of critically ill patients with sepsis. Clinical Science. 2012;122:133-42. doi: 10.1042/CS20110233.

19. Stana F, Vujovic M, Mayaki D, Leduc-Gaudet J-P, Leblanc P, Huck L, et al. Differential Regulation of the Autophagy and Proteasome Pathways in Skeletal Muscles in Sepsis: Critical Care Medicine. 2017;45:e971-9. doi: 10.1097/CCM.0000000000002520.

20. Chatre L, Verdonk F, Rocheteau P, Crochemore C, Chrétien F, Ricchetti M. A novel paradigm links mitochondrial dysfunction with muscle stem cell impairment in sepsis. Biochimica et Biophysica Acta (BBA) - Molecular Basis of Disease. 2017;1863:2546-53. doi: 10.1016/j.bbadis.2017.04.019.

21. Rocheteau P, Chatre L, Briand D, Mebarki M, Jouvion G, Bardon J, et al. Sepsis induces long-term metabolic and mitochondrial muscle stem cell dysfunction amenable by mesenchymal stem cell therapy. Nat Commun. 2015;6:10145. doi: 10.1038/ncomms10145.

22. Goossens C, Weckx R, Derde S, Dufour T, Vander Perre S, Pauwels L, et al. Adipose tissue protects against sepsis-induced muscle weakness in mice: from lipolysis to ketones. Crit Care. 2019;23:236. doi: 10.1186/s13054-019-2506-6.

23. Koutnik AP, Poff AM, Ward NP, DeBlasi JM, Soliven MA, Romero MA, et al. Ketone Bodies Attenuate Wasting in Models of Atrophy. Journal of Cachexia, Sarcopenia and Muscle. 2020;11:973-96. 
24. Demirel A, Li J, Morrow C, Barnes S, Jansen J, Gower B, et al. Evaluation of a ketogenic diet for improvement of neurological recovery in individuals with acute spinal cord injury: study protocol for a randomized controlled trial. Trials. 2020;21:372. doi: 10.1186/s13063-02004273-7. 
Figures

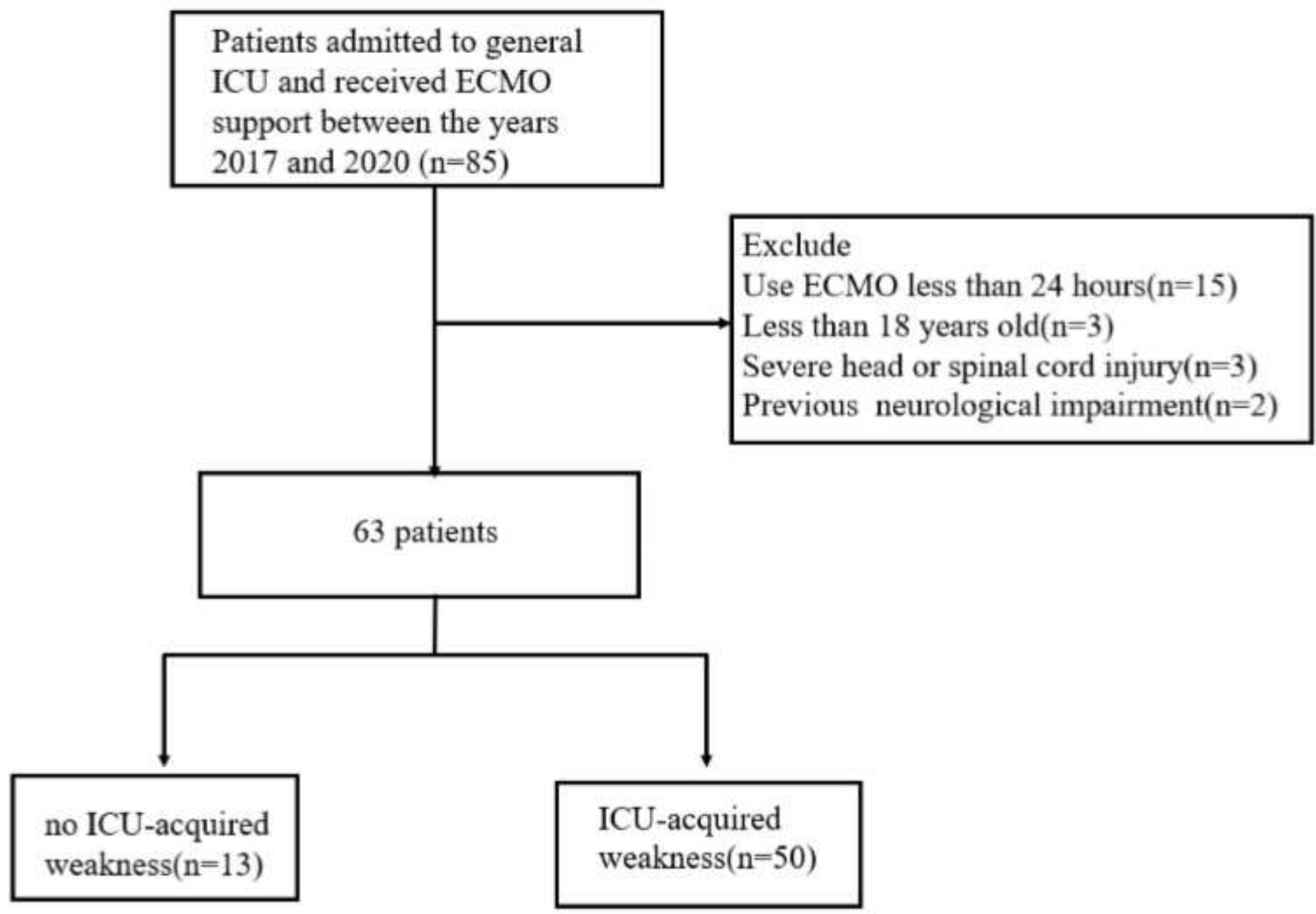

Figure 1

Flowchart of the study 


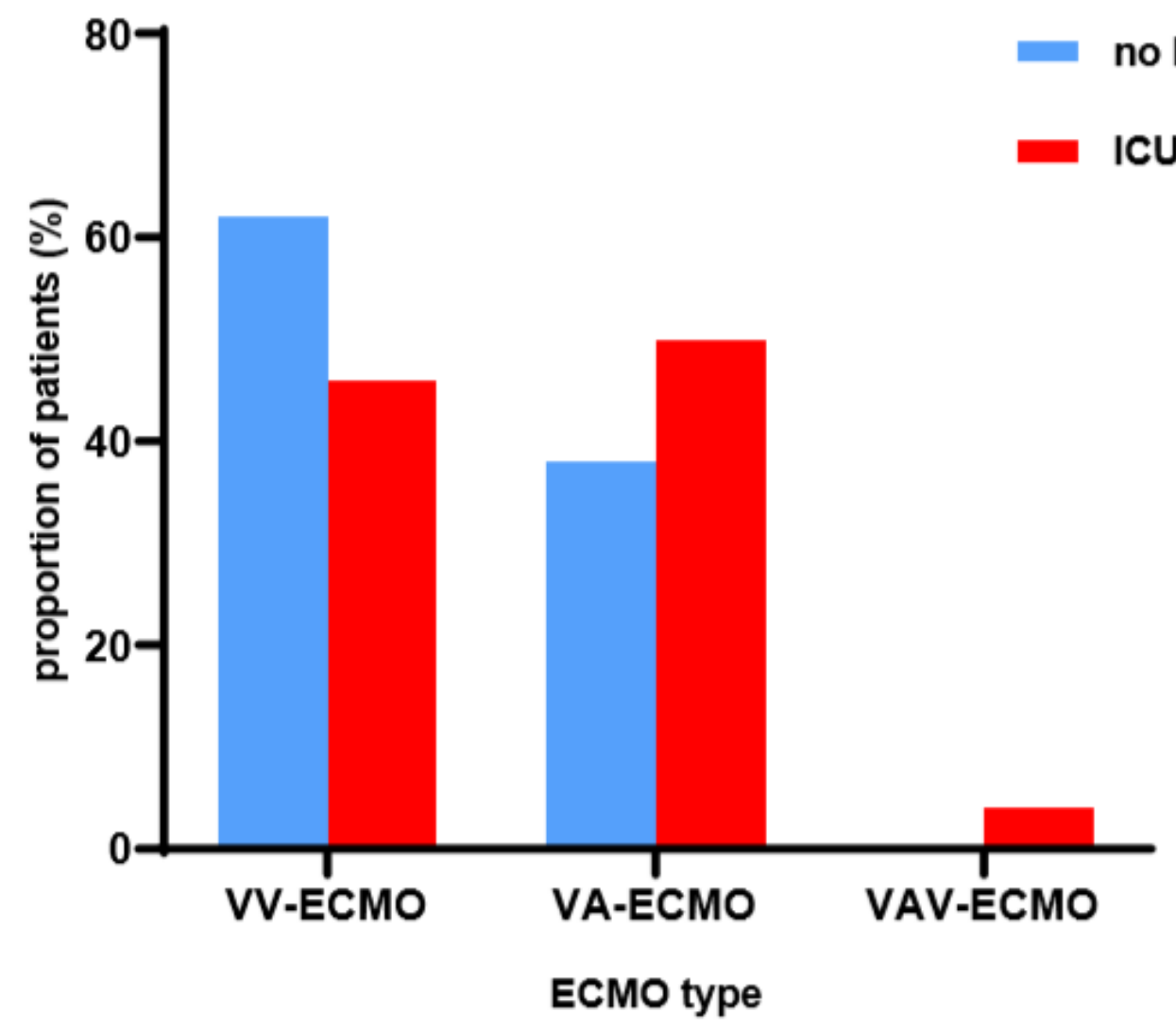

Figure 2

Users of different types of ECMO

\section{Supplementary Files}

This is a list of supplementary files associated with this preprint. Click to download.

- Table1Baselinecharacteristics.pdf

- Table2Therapydetails.pdf

- Table3ECMOmanagement.pdf

- Table4Logisticregressionanalysis.pdf 